Research Article

\title{
College English Flipped Classroom Teaching Model Based on Big Data and Deep Neural Networks
}

\author{
Heli Chang (iD \\ Department of Foreign Languages, Liaocheng University Dongchang College, Liaocheng 252000, Shandong, China \\ Correspondence should be addressed to Heli Chang; changheliliaocheng@163.com
}

Received 2 April 2021; Revised 23 April 2021; Accepted 5 May 2021; Published 25 May 2021

Academic Editor: Shah Nazir

Copyright $\odot 2021$ Heli Chang. This is an open access article distributed under the Creative Commons Attribution License, which permits unrestricted use, distribution, and reproduction in any medium, provided the original work is properly cited.

\begin{abstract}
With the rapid development of information technology, flipped classroom as a new type of mixed teaching mode relying on computer technology has changed the traditional teaching mode and formed a teaching process of "learning first and teaching later," and it has been used in many fields of teaching. Flipped classroom reverses the sequence of traditional teaching knowledge transfer and knowledge internalization and improves students' autonomy. However, it is still in the exploratory stage of the specific impact of the flipped classroom teaching model on college students' English autonomous learning ability. Therefore, this article proposes a novel college English flipped classroom teaching model based on big data and deep neural networks. The study has selected a total of 230 students in two classes of the second-year English major of a university as the research objects. Data are utilized to investigate the changes of the two groups of students' English autonomous learning ability and English academic performance, to explore the specific changes of college students' English autonomous learning ability and its influencing factors through interviews, and to predict and effectively analyze the weight of influencing factors through the deep neural network. This research enriches the theoretical research results of college students' English autonomous learning ability under the flipped classroom teaching model, provides reference for the cultivation of college students' English autonomous learning ability, and has certain reference significance for the optimization of the flipped classroom teaching model. The proposed research will support researchers and practitioners at college and university level.
\end{abstract}

\section{Introduction}

The traditional college English teaching model [1-3] deprives learners of English reading ability and communication ability, and it is difficult to tap learners' learning potential. In addition, teachers spend too much time in the classroom, and learners lack opportunities for language communication and exercise. In the traditional teacher management model, the teacher is the protagonist of the college English teaching work model [4]. The learners have not developed good self-study habits; in the classroom, the learners are still passive and mechanically taking notes. As a result, many learners' interest in English learning is not high, and the effect of English teaching activities is not ideal. In order to change these problems in English teaching, it is imperative to discover more effective English teaching models. The new curriculum standard advocates the improvement of students' autonomous learning ability and innovation ability. It does not require teachers to teach knowledge content comprehensively, but to allow learners to learn independently. This is an important teaching concept in flipped classroom education practice. This article believes that using flipped classroom teaching mode to design high school English reading classes can be a good solution, making teachers become knowledge leaders [5]. With the development of flipped classroom technology, researchers have applied the flipped classroom teaching model to various subjects in teaching, but few researchers have applied it to English reading, especially in high school English teaching, so I believe that flipped classroom should be applied. Research in college English teaching is very valuable [6].

Flipped classroom is a new teaching mode [7-10]; that is, teachers can use modern electronic data and information 
management technology to change the traditional English class into a need to distribute the learning materials to learners in advance, and the learners can design according to the teacher's education and teaching activities. The questions asked are studied independently before and after class. Teachers and students discuss with each other to solve difficult problems. It overturns the traditional forms of teaching and learning. To a large extent, "students listen and teachers speak" has been changed. Although there are already some problems in some high school education and teaching activities that require teachers to solve them through research and use of the flipped classroom teaching work model, there are still very few practices in English learning. In addition, the current flipped classroom is still based on platforms such as WeChat, which cannot guarantee learners' autonomous learning ability. The existing college English flipped classrooms cannot effectively solve these problems for us. Therefore, it is necessary to conduct some empirical research methods and use experiments to verify whether flipped classrooms have the ability to promote the development of English teaching [11].

Searching with the keywords of "flipped classroom" and "English teaching" indicated that there is a serious lack of research on college English teaching, and the design of the research content of English flipped classroom is also extremely incomplete, ignoring some system designs in the curriculum. In order to speed up the curriculum reform of college English education technology and improve the learning methods, skills, and class efficiency of English reading, it is essential to apply flipped classrooms to college English teaching practice activities [12].

This paper attempts to apply big data technology and deep neural network [13-16] technology to the college English flipped classroom teaching model and use this model to predict and analyze the factors affecting the quality of English flipped classrooms, which can further improve the teaching quality of flipped classrooms and become a flipped classroom. The paper also provides some valuable suggestions, including college English teaching design and research methods. The following are the main contributions points of this paper:

(i) This paper proposes a novel flipped classroom teaching model for college English based on big data and deep neural networks. First, it collects and sorts big data for flipped classrooms. Secondly, it uses deep neural networks to build a flipped classroom recommendation model to achieve personalized learning resource recommendation, which can improve the quality of college English flipped classroom teaching.

(ii) This paper combines the convolutional neural network and the gated recurrent unit to propose a college English flipped classroom prediction model. The model uses the convolutional neural network to automatically extract local features from the flipped classroom big data and is based on the learner's learning behavior. Related to timing, the model introduces a gated loop unit GRU, which enables the model to have good timing information extraction capabilities.

(iii) This paper also introduces an attention mechanism, which combines learner information and course information into the predictive model to improve the generalization ability of the predictive model.

The organization of the paper is given as follows. Section 2 briefly represents the related work to the proposed study. Section 3 of the paper shows the methodology section of the paper with in-depth details. Experiments and results of the paper are given in Section 4. The paper is concluded in Section 5 .

\section{Related Work}

The term "flipped classroom" was first used by American economist Rach Pratt in 2000, and its meaning refers to the integration of our traditional classroom from inside to outside of the classroom. Flipped classroom is to reverse the learning process in the traditional sense, complete the teaching of knowledge before class, and complete the internalization of knowledge in class. American scholars believe that flipped classroom refers to the flipping of the classroom of "teaching in class and homework after class." The idea is to flip the traditional learning process, complete the transfer of knowledge before class, and complete the internal knowledge in the classroom. In the process of transformation, flipped classroom is also called "reversal classroom." It is relative to the classroom teaching method of traditional Chinese culture. The traditional classroom teaching is the teaching of relevant knowledge by the teacher in the classroom, and the learners are in the class. Later, the internalization of knowledge can be achieved through homework and practice [17].

The definition of "flipped classroom" in this article is as follows: learners use instructional videos or electronic textbooks produced by teachers for autonomous learning before class and conduct interactive activities between teachers and students or peers, as well as answering questions and cooperating in the classroom. As a form of education and teaching, flipped classroom is different from the traditional teaching model of "teacher speaks, students listen." Instead, it allows learners to learn by watching the microvideo prepared by the teacher before class and summarize the content in the video. The content of the video mainly includes analysis of reading, grammatical points, background knowledge of reading materials, and related consolidation exercises. After completing the preclass microvideo study, learners can have a general understanding of the reading materials, better understand the subject and content of the reading materials, and clarify their own problems and deficiencies in the reading process. In the 
course of the classroom, the learners use the inquiry learning method to discuss each other, help each other to complete each other's problems, and exchange opinions so that the learners can draw lessons from the ideas of others. In addition, the learners are in the information. The communication platform can also provide better communication and discussion with teachers, which is extremely beneficial to learners. For some important and difficult points, learners can also review and consolidate knowledge through video review [18] and achieve the overall improvement of reading comprehension ability.

In flipped classroom education and teaching, the production of micro-videos is also extremely important. Learners need to summarize knowledge and key points by watching the videos themselves. After watching microvideos, learners can have a general understanding of English reading materials. The background knowledge mentioned in the video can also help high school learners to expand their professional knowledge and consolidate their practice so that they can better understand the main ideas and content of the reading materials and ask themselves clearly the possible questions in the reading process [19].

The traditional teaching mode of reading is teacher-led and learners passively accept the knowledge. Teachers keep lecturing and learners keep taking notes. As a result, learners are limited in learning knowledge due to teaching activities, so the reform of English teaching mode is imperative. Nowadays, flipped classroom has attracted wide attention. This teaching mode can highly improve the classroom education and teaching efficiency. In classroom activities, teachers and learners form a positive atmosphere of teaching and learning. Among learners, inquiry-based learning methods are used to discuss the difficult problems with each other and help each other to complete the exchange of views, so as to enable learners to learn from others' ideas and thus improve the quality of college English learning [20].

\section{Methodology}

In Figure 1, in the flipped classroom teaching model, teachers need to make microvideos before class to promote good learning for learners and allow learners to carry out targeted training in education and teaching. Learners can explore solutions through group cooperative learning. For these issues, the teacher will finally give feedback and comments.

The following sections briefly show the methodology of the paper.

\subsection{English Course Resource Recommendation}

3.1.1. Problem Description. How to realize the personalized learning resources recommended is essential to analyze the relationship between learners and learning resources to flip the classroom teaching content. Providing personalized course is the key to big data acquisition course and to effective analysis, data mining, and other processing means, eventually recommending appropriate English curriculum resources to learners. Personalized learning resources recommended model can make use of the history of the learners' learning resources for collecting information. The information study can be used as shown in Figure 2 in the history of the matrix. $R$ is for learning resources, $L$ represents the learner, the shaded part represents the learning resources learned by the learner, and the white blank part represents the learning resources not learned. To solve the problem is how to implement personalized learning resources through the historical information matrix recommendations; namely, the new learning resources in the recommended resources can meet the demand of learners.

3.1.2. Recommended Model. According to the personalized learning resources, a description of the problem is recommended. This paper constructs the recommendation model. This model can be summed up in two processes, namely, training process and recommendations, as shown in Figure 3. The training process includes learning platform data processing, such as algorithm design process, based on deep learning algorithm, optimizing the depth of the neural network [21-24], and more efficient and reasonable training process. The recommended process recommended models which are obtained by training process, obtaining the personalized learning resources. The recommendation model can be divided into three levels, in which the first and second levels belong to the training process, and the third level is the personalized learning resource recommendation process. In the first stage, in order to make the deep neural network work, it is necessary to obtain its input and output. For the input, this paper designs a feature selection model based on MIFS to describe and process the historical learning data. For output, the learner-resource bipartite graph association model is constructed, and the conditions of whether learning resources are recommended or not are defined. In the second stage, the depth of the neural network learning model is optimized through the first level of input and output; in order to address the problem of customized recommendation, a variety of neural network strategies are modeled and optimized, and a recommended model is available through the training phase of complex learning tools. The third level is where qualified learning resources can test data as input to the suggested model and can be recommended to adhere to the learners' high accuracy of customized learning resources.

3.1.3. Feature Selection Module. The attributes of learners and learning resources have diversified characteristics. There are many factors that affect learners' choice of learning resources, which may include characteristics such as gender, majors, learning goals, content preferences, learning styles, cognitive levels, and learning motivations; on the other hand, learning resources may have inherent attributes such as resource styles and interaction methods. Therefore, it is necessary to find the relationship between learners and resources among many features and establish a feature selection model to complete the input process of the recommendation method. 


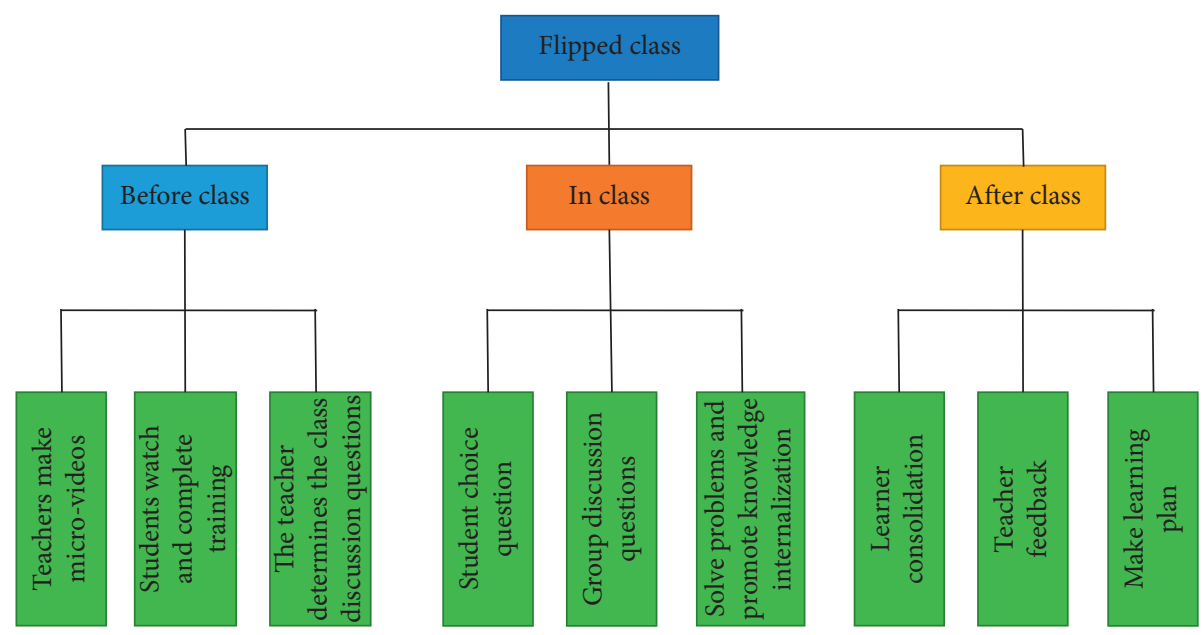

FIGURE 1: Teaching mode of college English flipped class.
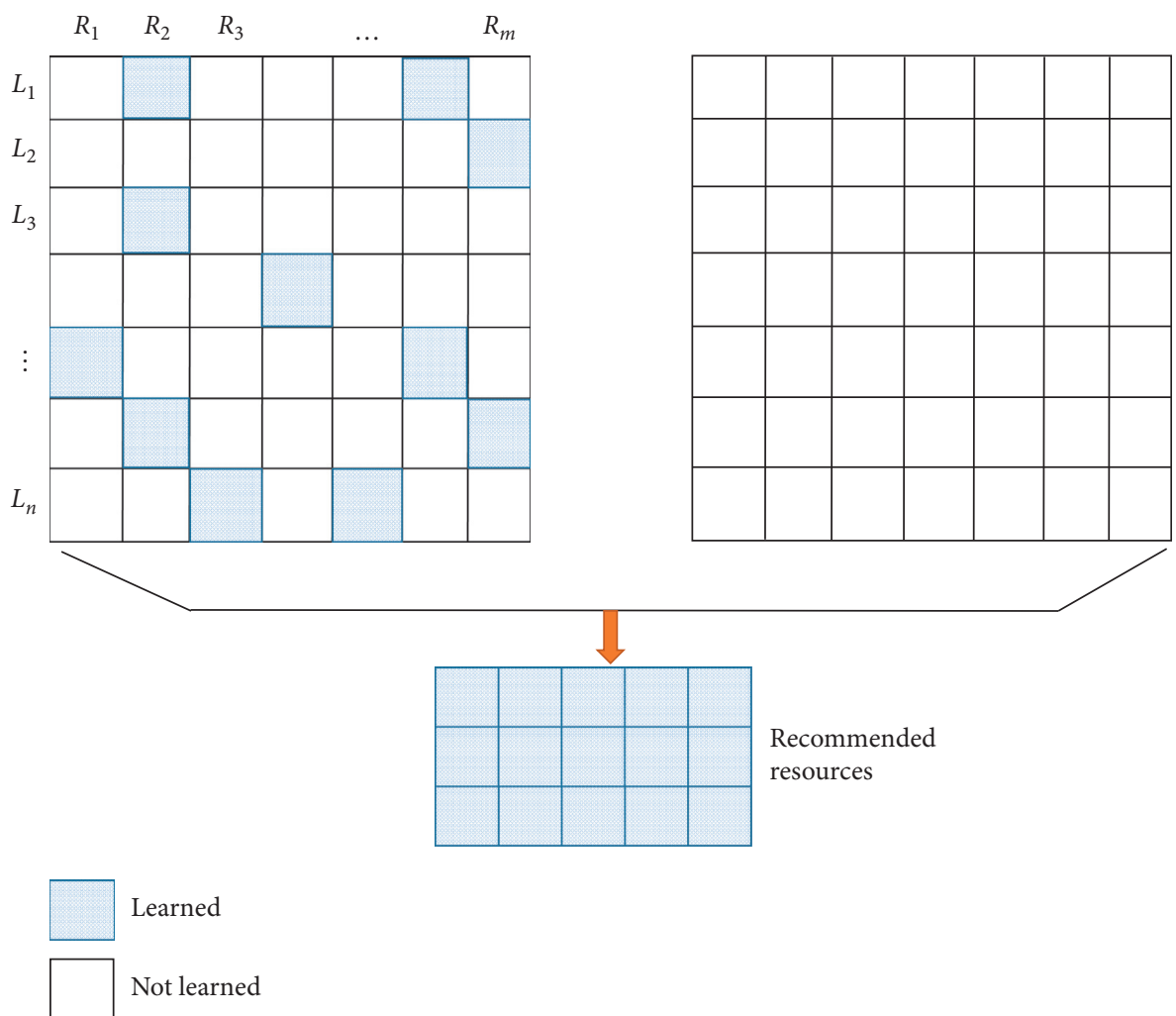

FIGURE 2: Schematic diagram of the recommendation problem of college English personalized learning resources.

The filtering feature selection method can usually directly use the performance of the training data to evaluate the features, and it has nothing to do with the subsequent algorithm; the speed is faster, and the relationship between the actual data is selected, so the method based on mutual information feature selection (MIFS) is selected. The information describes the strength of the association between two random attributes or features and judges the correlation between a single feature and the target category, thereby reducing the redundancy of feature dimensions. Figure 4 shows the MIFS-based feature selection model.
In the MIFS-based feature selection method, the information measurement evaluation function is very important. Although the function has various forms, the purpose is to select the feature subset that has the greatest correlation with the category. The generalized information measurement evaluation function can be expressed as

$$
J(f) \alpha \times g(C, f, S)-\delta,
$$

where $S$ is the selected feature, $f$ is the candidate feature, $C$ is the category, function $g(C, f, S)$ is the amount of 


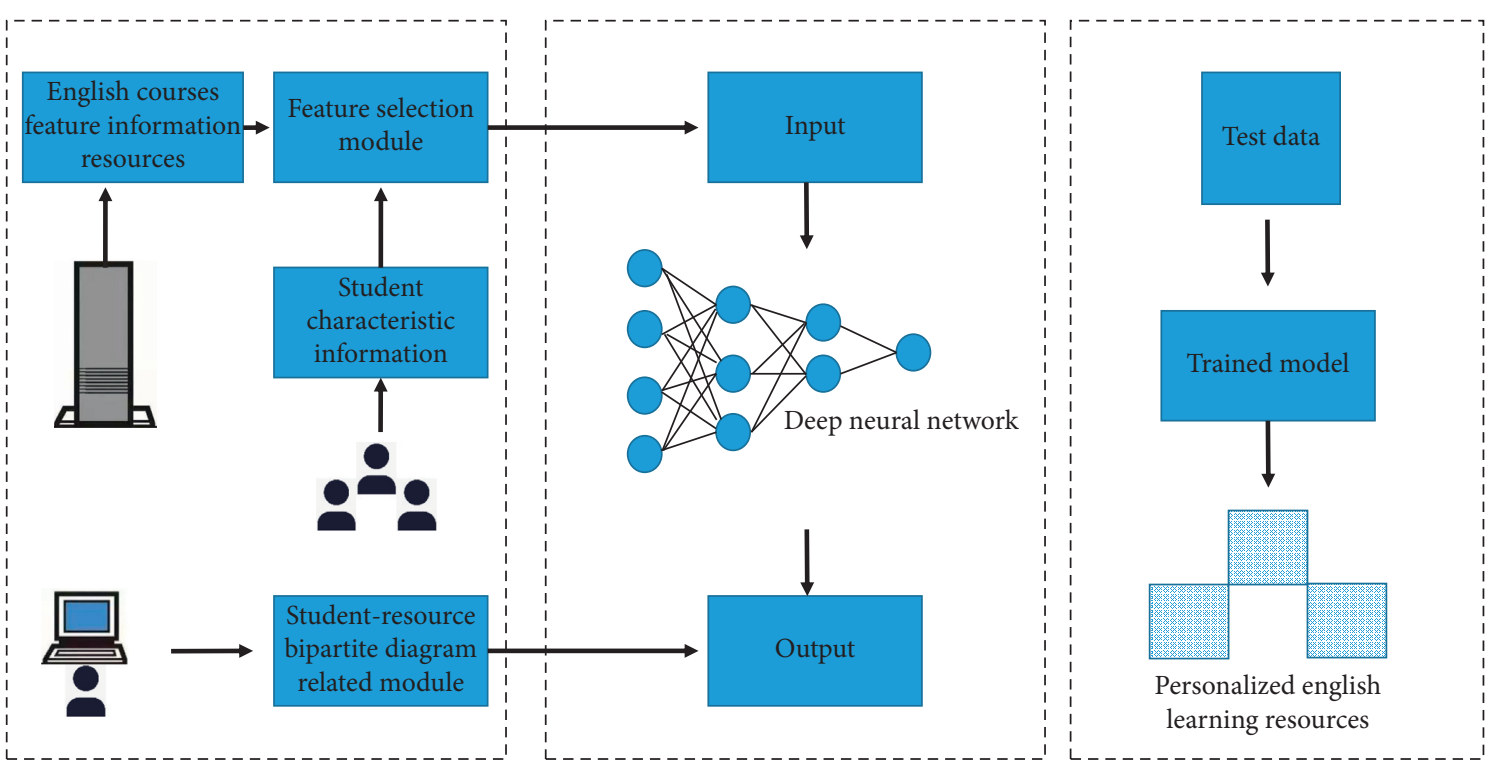

FIgURE 3: English resource recommendation model.

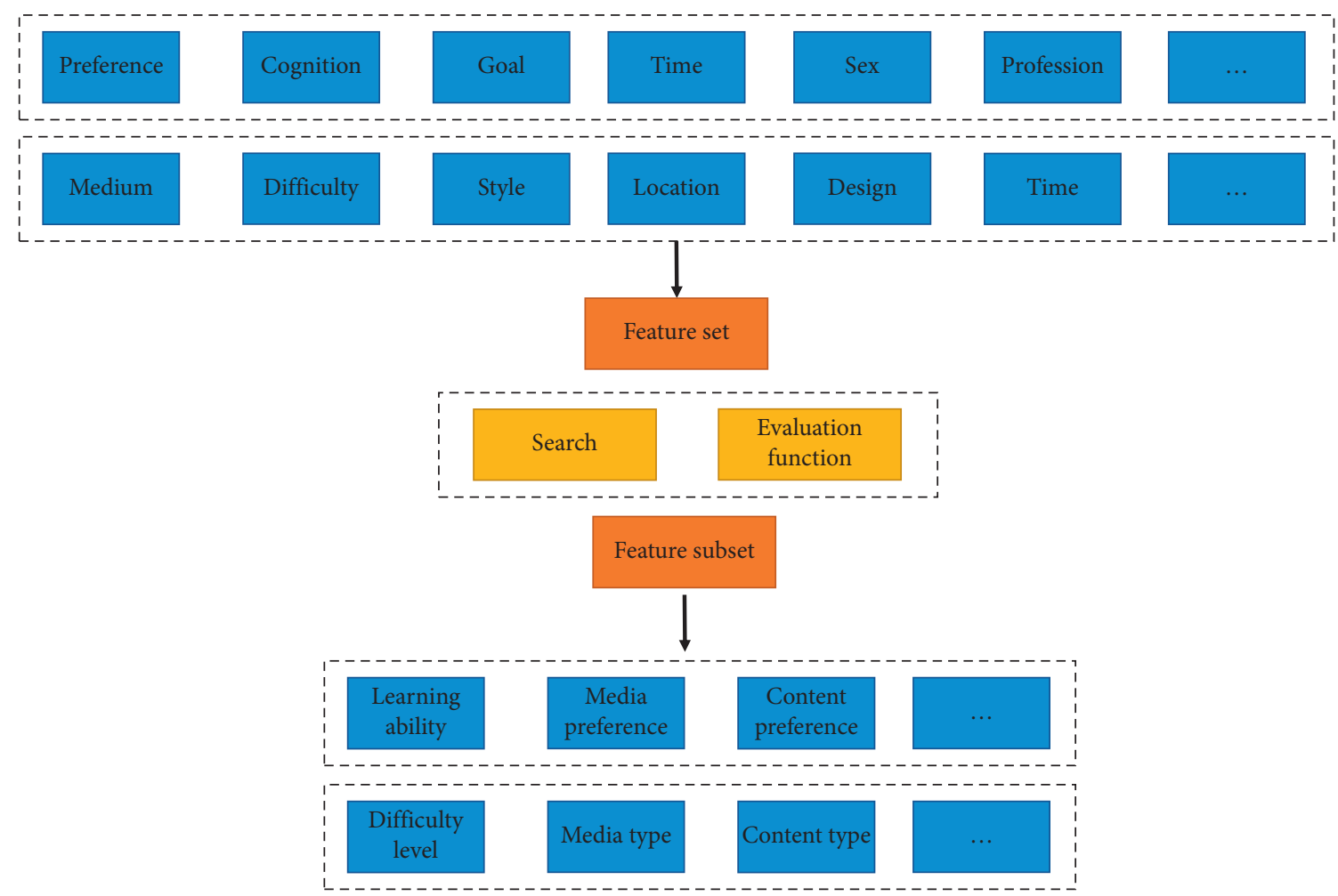

Figure 4: Feature selection model.

information between $C, f$, and $S$, that is, between the selected feature and the category after adding the candidate feature, $\alpha$ is the adjustment coefficient, used to adjust the degree of information brought by the addition of $f$, and a is the penalty factor, used for the degree of redundancy that $f$ brings to $S$. Therefore, the simplest and most intuitive information measurement evaluation function can be

$$
J(f)=g(C, f),
$$

where $g$ is mutual information and $\beta$ is the regulation coefficient. Then, all the candidate features are directly calculated as the evaluation function to prevent excessive redundancy in the preprocessing. Considering the correlation between features, $f$ is punished by the correlation 
between feature $f$ and the selected single feature $s$, and the final evaluation function can be expressed as

$$
J(f)=g(C, f)-\beta \sum_{s \in S} g(s, f) .
$$

It is particularly important to determine the learners' preference for the features of learning resources. The selected features indicate some features that will affect learners' choice of resources, such as the knowledge content and learning duration of resources. These features can be determined according to the sorting of known studies or the investigation methods. On behalf of the temporary, feature candidates are unsure if learners can learn the characteristics of some specific tools, such as learners' age, professional history, gender, and category of the selected feature extraction, which will be used to calculate the selected features and candidate information correlation. The construction of final evaluation function is to add larger correlation feature candidates, determine the candidate characteristics' influence on the evaluation results, and filter out some redundant features. The purpose is to effectively alleviate the late use of data for deep learning training workload.

\subsubsection{Student-Resource Bipartite Graph Correlation Model.} This paper presents a bipartite graph association model of learner-resource and defines the learner set as $L=\left\{l_{1}, l_{2}, \ldots, l_{m}\right\}$. The resource set is $R=\left\{r_{1}, r_{2}, \ldots, r_{n}\right\}$. So can get a set by the learners and resource collections of binary relation matrix $X_{m \times n}$, row vector on behalf of the learners, column represents the learners to learn about the resources, if $X_{m \times n}=1$, this indicates the learners to learn about the resources, if $X_{m \times n}=0$ is not learned. In fact, this simple unitary processing cannot objectively reflect the degree of learners' awareness of the learning resources, so learning the frequency of a particular resource cannot be ignored.

$$
X_{m \times n}=\left[\begin{array}{cccc}
X_{11} & X_{21} & \cdots & X_{m 1} \\
X_{12} & X_{22} & \cdots & X_{m 2} \\
\vdots & \vdots & \cdots & \vdots \\
X_{1 n} & X_{2 n} & \cdots & X_{m n}
\end{array}\right] .
$$

Learners' learning frequency of learning resources can reflect different degrees of preference. The average frequency of resource learning can be defined as

$$
\bar{r}\left(l_{i}\right)=\frac{\sum_{j=1}^{j} k\left(l_{i}, r_{j}\right)}{n\left(l_{i}\right)},
$$

where $\bar{r}\left(l_{i}\right)$ represents the average number of times $l_{i}$ of using learning resources, $n\left(l_{i}\right)$ represents the number of learning resources $l_{i}$ used, and $\sum_{j=1}^{j} k\left(l_{i}, r_{j}\right)$ represents the total number of learning resources. The average number of times of using learning resources $R\left(L_{i}\right)$ is taken as the critical value, which is the classification analysis of the recommendation model.

$$
\begin{cases}k \geq \bar{r}\left(l_{i}\right) & \text { recommend } \\ k \leq \bar{r}\left(l_{i}\right) & \text { not recommended }\end{cases}
$$

\subsection{Deep Neural Network}

3.2.1. Attention Mechanism. The input information of the model can be represented by set $X=\left[X^{1}, X^{2}, \ldots, X^{T}\right]$, and each element of the set $X$ represents the input information at a certain moment. The index position of the selected information can be represented by the attention variable $z \in[1, T]$. When $z=t$, it means that the $t$-th information is selected in the input information set $X$. Given a task-related query vector $q$ and input information $X$, first calculate the attention distribution (probability distribution) $a_{t}$, which represents the $t$ input information:

$$
a_{t}=\frac{\exp \left(X_{t}^{T} W q\right)}{\sum_{1 \leq t \leq T} \exp \left(X_{t}^{T} W q\right)} .
$$

The attention distribution can be interpreted as the degree to which the $t$-th information receives attention when the context queries $q$. The input information is encoded by a "soft" information selection mechanism, which is called the soft attention mechanism (as shown in Figure 5):

$$
\text { attention }=\sum_{t=1}^{N} a_{t} X_{t} .
$$

The main role of the attention mechanism is to effectively reduce the dimension size of the input data of the model based on the corresponding network structure without changing the original position of the input data. In this way, the design defect of the traditional codec is avoided, and the input data is not limited to a fixed length, which reduces the information loss of the input data.

3.3. GRU. As a kind of LSTM improved recursive neural network, GRU gated circulation unit has a simpler gate structure, which reduces a lot of matrix multiplication computation in the operation of neural network. At the same time, GRU networks can make better use of historical data and learn about the long-term dependence of information. The activation function of the neural network is responsible for mapping the input of the neuron to the output, which increases the nonlinear ability of the neural network model. The choice of activation function directly affects the performance of GRU network and other neural network structures. At present, activation functions commonly used in deep learning include Sigmoid function and tank function belonging to the Sigmoid system and ReLU function of the Modifying Linear Unit (ReLU) system.

But the ReLU function also has drawbacks. Firstly, in the process of neural network training, part of the input will enter the hard saturated region of ReLU function so that some neurons will never be activated by any data again, resulting in neuronal necrosis. Secondly, the output of the ReLU function has no negative value, which will cause the 


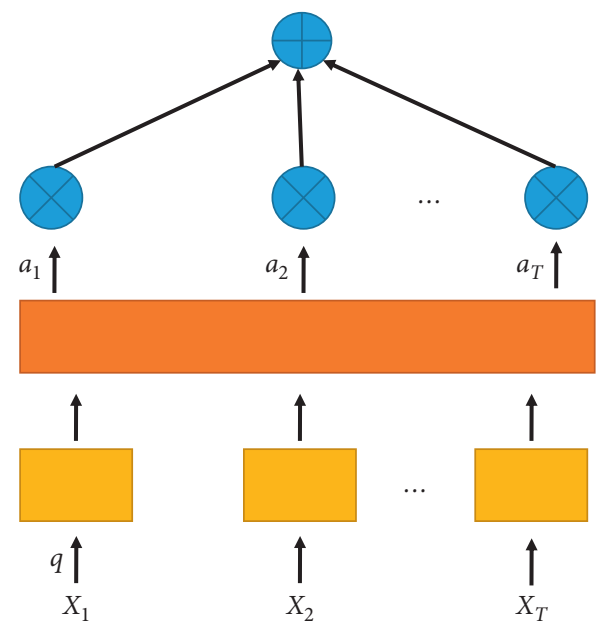

Figure 5: Schematic diagram of soft attention mechanism.

mean shift phenomenon of the output which is not the zero mean value. Mean shift is easy to cause the neurons in the lower layer to get the nonzero mean signal output by the upper layer as input, which makes it difficult to calculate the weight matrix $\mathrm{W}$ of the network. Therefore, this paper uses ELU to optimize the performance of the neural network. The ELU function expression and the derivative expression are as follows:

$$
f(x)\left\{\begin{array}{ll}
a\left(e^{x}-1\right) & x \leq 0 \\
x & x>0
\end{array}, f(x) \begin{array}{ll}
f(x)+a & x \leq 0 \\
1 & x>0
\end{array} .\right.
$$

\section{Experiments and Results}

4.1. Experimental Environment. Since the experiment in this article needs to train a deep neural network, the scale is large, the structure is more complex, and the calculation scale is large. The programming language used is Python, the version is 3.6.5, the deep learning framework used is Pytorch 0.4 , the IDE for program deployment is Pycharm, and all experiments are conducted in the same environment. All our experiments have been conducted on a desktop PC with an Intel Core i7-8700 processor and an NVIDIA GeForce GTX 1080 GPU.

4.2. Experimental Setup. To verify that the personalized learning resources recommended by the method in this paper meet the needs of learners, a series of experiments were conducted. Experimental data includes not only learning resource data, but also the historical data of learners' learning. In the existing public data sets, such as EDX, World UC, and other data sets, it provides dozens of attributes, including course data, learner information, and learner behavior data. This paper also collects the flipped classroom big data from two classes of an English major in a university. In this experiment, the initial learning rate is set to 0.001 , and after 15,000 and 20,000 iterations, the learning rate becomes one-tenth of the original, and 30 training data sets are fed each time.
4.3. Evaluation Index. Accuracy rate $(P)$, recall rate $(R)$, and $F 1$-score value $(F)$ were used for evaluation in this paper. The calculation equation is as follows:

$$
\begin{aligned}
& P=\frac{\mathrm{TP}}{\mathrm{TP}+\mathrm{FP}}, \\
& R=\frac{\mathrm{TP}}{\mathrm{TP}+\mathrm{FN}}, \\
& F=\frac{2 \times P \times R}{P+R} .
\end{aligned}
$$

4.4. Experimental Results. Table 1 is a sample used in this paper. It can be seen from the experimental results with the change of the sample size data sets that the algorithm precision $(P)$ and the recall rate $(R)$ will follow but are at a higher value. This paper that is constructed for personalized learning resources recommended problem model, and using machine learning algorithm is feasible and effective, with the classification of evaluation indexes. The $P$ and $R$ often cannot be in good value, such that sample $1 R$ value of the SVM algorithm is higher, but the $P$ value is smaller, not set at this time from an indicator to judge its performance, therefore to $F 1$-score values $(F)$ as recommended comprehensive evaluation index of performance. As you can see, the depth of the neural network designed (DE) on the $F$ value is superior to the other traditional machine learning algorithms and has high value. In order to verify the method adapted to different scale data sets, combined with Table 2, it can be seen as the cumulative learning more and long time, in different times, due to the learners or other factors affect the situation. All kinds of indexes will change, but the trend is still good, as shown in Figures 6-8. For different samples along with the increase in quantity or learning time, three of the classification indexes will increase, studying the records. It is conducive to the correlation analysis between learners and learning resources and provides recommendation performance. From the algorithm comparison level, it can be seen that the algorithm based on deep neural network model is 
TABLE 1: Data sample descriptions.

\begin{tabular}{lccc}
\hline & Sample 1 & Sample 2 & Sample 3 \\
\hline Number of learners & 50 & 80 & 100 \\
Time (days) & 15 & 15 & 30 \\
Learn the number of records & 7528 & 12015 & 30021 \\
\hline
\end{tabular}

TABLe 2: Experimental results.

\begin{tabular}{lccccccrrr}
\hline \multirow{2}{*}{ Methods } & \multicolumn{3}{c}{ Sample 1 } & \multicolumn{3}{c}{ Sample 2 } & \multicolumn{2}{c}{ Sample 3 } \\
& $P$ & $R$ & $F$ & $P$ & $R$ & $F$ & $P$ \\
\hline DT & 0.38 & 0.53 & 0.44 & 0.45 & 0.53 & 0.4 & 0.59 & 0.60 \\
SVM & 0.45 & 0.53 & 0.48 & 0.54 & 0.55 & 0.54 & 0.66 & 0.68 \\
KNN & 0.57 & 0.57 & 0.57 & 0.51 & 0.56 & 0.70 & 0.74 & 0.76 \\
Ours & $\mathbf{0 . 6 2}$ & $\mathbf{0 . 6 1}$ & $\mathbf{0 . 7 1}$ & $\mathbf{0 . 6 0}$ & $\mathbf{0 . 6 5}$ & $\mathbf{0 . 7 5}$ & $\mathbf{0 . 7 8}$ & $\mathbf{0 . 7 8}$ & 0.75 \\
\hline
\end{tabular}

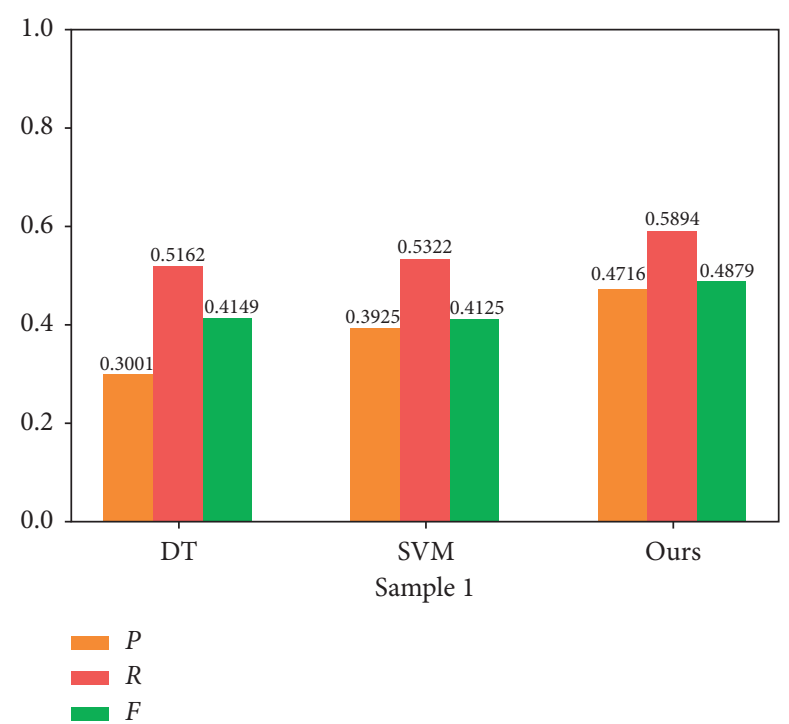

FIgURE 6: Histogram of evaluation index results for sample 1.

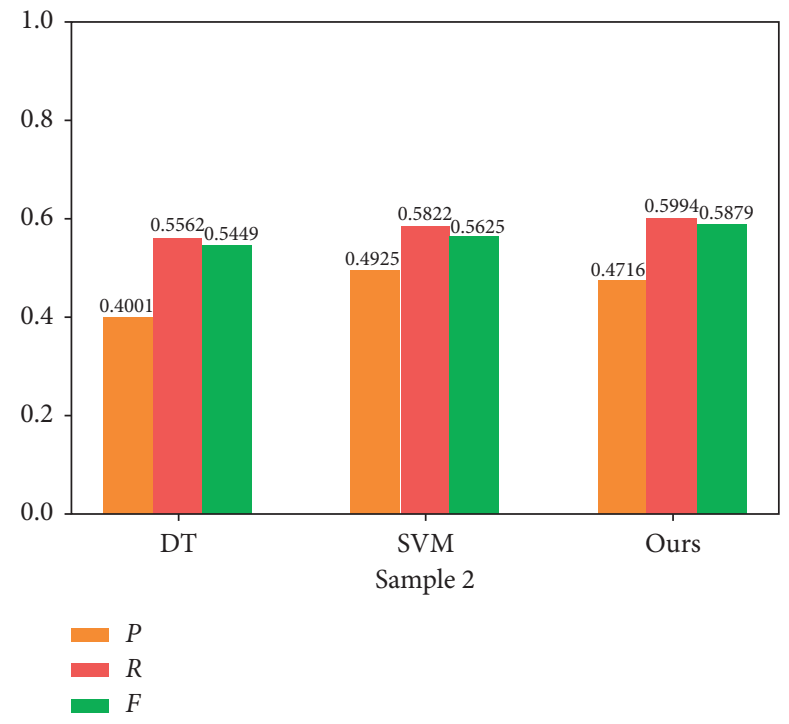

FIgURE 7: Histogram of evaluation index results for sample 2. 


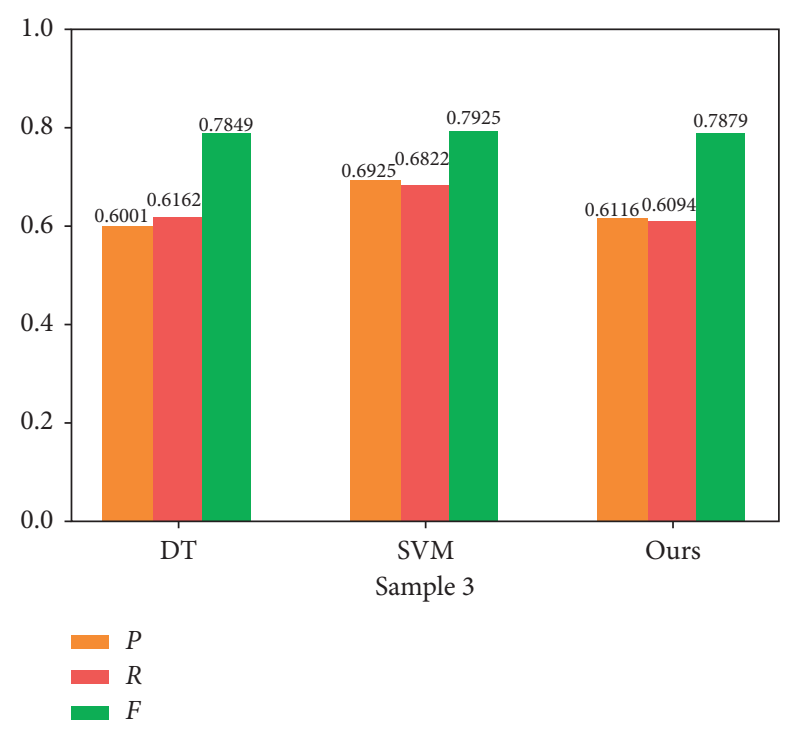

Figure 8: Histogram of evaluation index results for sample 3.

better than other algorithms, indicating that the method proposed in this paper is consistent with and targeted to the practical problem of learning resource recommendation.

It can be clearly seen that the error between the predicted result and the actual result is the smallest, indicating that the improved deep neural network has good adaptability in predicting the frequency of learners' learning resources and shows better performance than other algorithms. This is because, with the increase in learning behavior records, learners' learning rules for resources become more and more obvious. The error judgment of regression analysis is helpful in predicting learners' interests and can improve the teaching quality of college English flipped classrooms.

\section{Conclusion}

This paper proposes a novel college English flipped classroom teaching model based on big data and deep neural networks. The study selects a total of 230 students in two classes of the second-year English major of a university as the research objects. Data are utilized to investigate the changes of the two groups of students' English autonomous learning ability and English academic performance, to explore the specific changes of college students' English autonomous learning ability and its influencing factors through interviews, and to predict and effectively analyze the weight of influencing factors through the deep neural network. This research enriches the theoretical research results of college students' English autonomous learning ability under the flipped classroom teaching model, provides reference for the cultivation of college students' English autonomous learning ability, and has certain reference significance for the optimization of the flipped classroom teaching model.

\section{Data Availability}

The data used to support the findings of this study are included within the article.

\section{Conflicts of Interest}

The author declares no conflicts of interest.

\section{References}

[1] F. Zhang, "Quality-improving strategies of college english teaching based on microlesson and flipped classroom," English Language Teaching, vol. 10, no. 5, pp. 243-249, 2017.

[2] D. Qiu, "A study on english teaching model under the background of big data," Journal of Physics: Conference Series, vol. 1744, no. 3, Article ID 032061, 2021.

[3] D. Zhang and X. Wang, "The effects of the call model on college english reading teaching," International Journal of Emerging Technologies in Learning, vol. 12, no. 12, pp. 24-34, 2017.

[4] H. Zhang, S.-J. Jin, and S.-Z. Du, "Developing a curriculum model of English teaching for master's degree nursing education in a chinese medicine university," International Journal of Nursing Sciences, vol. 7, no. 1, pp. 99-104, 2020.

[5] M. Wiesenberg and R. Tench, "Deep strategic mediatization: organizational leaders' knowledge and usage of social bots in an era of disinformation," International Journal of Information Management, vol. 51, p. 10204, 2020.

[6] N. Guan, J. Song, and D. Li, "On the advantages of computer multimedia-aided english teaching," Procedia Computer Science, vol. 131, pp. 727-732, 2018.

[7] G. Akçayır and M. Akçayır, "The flipped classroom: a review of its advantages and challenges," Computers \& Education, vol. 126, pp. 334-345, 2018.

[8] K. F. Hew and C. K. Lo, "Flipped classroom improves student learning in health professions education: a meta-analysis," BMC Medical Education, vol. 18, no. 1, pp. 1-12, 2018.

[9] P. Strelan, A. Osborn, and E. Palmer, "The flipped classroom: a meta-analysis of effects on student performance across disciplines and education levels," Educational Research Review, vol. 30, Article ID 100314, 2020.

[10] Z. Turan and B. Akdag-Cimen, "Flipped classroom in english language teaching: a systematic review," Computer Assisted Language Learning, vol. 33, no. 5-6, pp. 590-606, 2020. 
[11] G.-Z. Liu, J.-Y. Chen, and G.-J. Hwang, "Mobile-based collaborative learning in the fitness center: a case study on the development of english listening comprehension with a context-aware application," British Journal of Educational Technology, vol. 49, no. 2, pp. 305-320, 2018.

[12] L. Shi, J. Delahunty, and X. Gao, "Constraints preventing Chinese EFL teachers from putting their stated beliefs into teaching practice," Professional Development in Education, vol. 45, no. 5, pp. 774-789, 2019.

[13] X. Ning, Y. Wang, W. Tian, L. Liu, and W. Cai, “A biomimetic covering learning method based on principle of homology continuity," ASP Transactions on Pattern Recognition and Intelligent Systems, vol. 1, no. 1, pp. 9-16, 2021.

[14] L. Zhang, X. Wang, X. Dong, L. Sun, W. Cai, and X. Ning, "Finger vein image enhancement based on guided triGaussian filters," ASP Transactions on Pattern Recognition and Intelligent Systems, vol. 1, no. 1, pp. 17-23, 2021.

[15] S. Li, X. Ning, L. Yu et al., "Multi-angle head pose classification when wearing the mask for face recognition under the COVID-19 coronavirus epidemic," in Proceedings of the 2020 International Conference on High Performance Big Data and Intelligent Systems (HPBD\&IS), pp. 1-5, IEEE, Shenzhen, China, May 2020.

[16] W. Cai, B. Liu, Z. Wei, M. Li, and J. Kan, “TARDB-Net: tripleattention guided residual dense and BiLSTM networks for hyperspectral image classification," Multimedia Tools and Applications, vol. 80, no. 7, pp. 11291-11312, 2021.

[17] L. Buckingham and R. S. Alpaslan, "Promoting speaking proficiency and willingness to communicate in Turkish young learners of english through asynchronous computer-mediated practice," System, vol. 65, pp. 25-37, 2017.

[18] S. Franceschini, P. Trevisan, L. Ronconi et al., "Action video games improve reading abilities and visual-to-auditory attentional shifting in english-speaking children with dyslexia," Scientific Reports, vol. 7, no. 1, pp. 1-12, 2017.

[19] N. Madikiza, M. P. Cekiso, B. P. Tshotsho, and N. Landa, "Analysing english first additional language teachers' understanding and implementation of reading strategies," Reading \& Writing, vol. 9, no. 1, pp. 1-10, 2018.

[20] D. Zou, "Research on college English teaching model based on multimedia and network," in Proceedings of the 3rd International Conference on Social Science and Technology Education, Wuhan, China, April 2017.

[21] X. Ning, W. Li, and J. Xu, "The principle of homology continuity and geometrical covering learning for pattern recognition," International Journal of Pattern Recognition and Artificial Intelligence, vol. 32, no. 12, Article ID 1850042, 2018.

[22] X. Zhang, Y. Yang, Z. Li, X. Ning, Y. Qin, and W. Cai, “An improved encoder-decoder network based on strip pool method applied to segmentation of farmland vacancy field," Entropy, vol. 23, no. 4, p. 435, 2021.

[23] Z. L. Yang, S. Y. Zhang, Y. T. Hu, Z. W. Hu, and Y. F. Huang, "VAE-Stega: linguistic steganography based on variational auto-encoder," IEEE Transactions on Information Forensics and Security, vol. 16, pp. 880-895, 2020.

[24] X. Ning, X. Wang, S. Xu et al., "A review of research on cotraining," in Concurrency and Computation: Practice and ExperienceJohn Wiley \& Sons, Hoboken, NJ, USA, 2021. 